\title{
A rapid method for staining inclusions of Chlamydia psittaci and Chlamydia trachomatis
}

\author{
RM WOODLAND, JAYSHREE MALAM, S DAROUGAR \\ From the Sub-department of Virology, Institute of Ophthalmology, Judd Street, London WC1H $9 Q S$
}

SUMmARY A new staining method was developed for the detection of inclusions of Chlamydia psittaci and Chlamydia trachomatis inclusions in cell cultures. Using a combination of methyl green and neutral red stains and washing at $\mathrm{pH} 5 \cdot 0$, inclusions were stained red while cell cytoplasm was pale pink and cell nuclei were pale green. The method was significantly better than Giemsa staining and comparable to immunofluorescence for detecting $C$ psittaci inclusions. Its sensitivity for detectin $C$ trachomatis inclusions by dark field microscopy was similar to that of Giemsa staining.

A variety of staining methods have been used to visualise chlamydial inclusions in cell culture monolayers and conjunctival scrapings. ${ }^{12}$ Giemsa stain, combined with dark field illumination, has been commonly used to detect Chlamydia trachomatis inclusions in cell culture monolayers, but this has not proved to be satisfactory for detecting Chlamydia psittaci inclusions. ${ }^{34}$ While such inclusions can be seen by bright field microscopy, the contrast is poor and they can be difficult to detect by low power microscopy. Iodine stain ${ }^{5}$ and carmine stain $^{6}$ detect the glycogen matrix of inclusions and are therefore only of use for mature $C$ trachomatis inclusions. Immunochemical staining methods, such as immunofluorescence (FA) and immunoperoxidase, ${ }^{4}$ are sensitive and reliable but require the use of expensive reagents. A methylene blue staining method for detecting ewe abortion agent ( $C$ psittaci) inclusions has been described, ${ }^{3}$ but its efficacy for other chlamydiae is not known.

In our laboratory, studies of human $C$ psittaci infections and of animal diseases caused by the agents of feline keratoconjunctivitis (FKC) and guineapig inclusion conjunctivitis (GPIC), which are both members of $C$ psittaci, as models for the study of human chlamydial infections, led to the need for a reliable, practical and inexpensive method by which inclusions produced by these organisms could be detected in cell culture monolayers. A new simple staining method, which uses methyl green and neutral red (MG-NR), has been developed for staining inclusions of both species of chlamydiae. Its sensitivity was compared with the sensitivities of the standard method of Giemsa staining, the

Accepted for publication 18 November 1981 methylene blue method, and immunofluorescence staining (FA) for detecting $C$ psittaci and $C$ trachomatis inclusions.

\section{Material and methods}

CHLAMYDIAL ISOLATES

The following $C$ psittaci agents were used to infect McCoy cell monolayers: feline keratoconjunctivitis agent (FKC) strain FKC/Ps/1/IOL-457/O; guinea pig inclusion conjunctivitis agent (GPIC) strain A10; meningopneumonitis agent (MPV) strain Cal-10; ewe abortion agent (EA); a human genital $C$ psittaci isolate-strain $33 \mathrm{~L}$; a human ocular $C$ psittaci isolate-strain IOL-395; and an atypical iodine-negative chlamydial agent-strain IOL-207.7 In addition, an oculogenital $C$ trachomatis agent, strain TRIC/D/USA-Cal/Cal-15/ON, was used.

\section{CLINICAL SPECIMENS}

Conjunctival and urethral swabbings were collected from guinea pigs which had been inoculated with GPIC agent or FKC agent and from rabbits which had been inoculated with FKC agent as part of another investigation. These swabbings were stored at $-70^{\circ} \mathrm{C}$ until inoculated on to McCoy cell monolayers.

\section{CELL CULTURE INOCULATION}

Laboratory isolates and animal specimens were cultured in cycloheximide-treated McCoy cells. ${ }^{8}$ The inoculated monolayers were incubated for $48 \mathrm{~h}$ in the case of FKC agent and $72 \mathrm{~h}$ for the other chlamydiae. They were then fixed in absolute methyl alcohol for $10 \mathrm{~min}$ and stained using either methyl 
green-neutral red (MG-NR), Giemsa, $0.5 \%$ methylene blue solution, ${ }^{3}$ or immunofluorescence (FA). ${ }^{4}$

THE MG-NR STAINING METHOD

Stock solutions of methyl green and neutral red were prepared as follows:

An aqueous solution ( $1 \% \mathrm{wt} / \mathrm{vol})$ of methyl green (Raymond A Lamb, 6 Sunbeam Road, London NW10) was prepared and extracted several times with chloroform until all traces of blue dye were removed. A neutral red solution $(0 \cdot 1 \% \mathrm{wt} / \mathrm{vol})$ was prepared by dissolving one gram of neutral red (GT Gurr, BDH, Poole, Dorset) and $2 \mathrm{ml}$ of $1 \%$ acetic acid in one litre of distilled water. The resulting solution was filtered immediately. These stock solutions have been stored at room temperature for several months with no apparent deterioration.

For use, a mixture consisting of nine parts methyl green solution and one part neutral red solution was prepared. This was usually used immediately but satisfactory staining has been achieved using mixtures which had been prepared and stored for at least two weeks.

Fixed cell monolayers were washed with a pH 5.0 buffer solution and then stained for $10 \mathrm{~min}$ in the methyl green-neutral red mixture. The monolayers were then washed in $\mathrm{pH} 5.0$ buffer solution for $10 \mathrm{~min}$, air-dried, and mounted in Gurrs' 'Uvinert' mountant (BHD, Poole, Dorset). Cell nuclei stained pale blue-green, cytoplasm was very pale pink, and inclusions were stained red.

\section{EXAMINATION OF STAINED MONOLAYERS}

Giemsa, methylene blue and MG-NR stained monolayers were examined by normal bright field microscopy or by dark field illumination. Immunofluorescence-stained monolayers were examined using a Zeiss fluorescence microscope fitted with an epi-illumination system.

\section{DESIGN OF EXPERIMENTS}

To compare the Giemsa, methylene blue and MGNR staining methods, each laboratory isolate was inoculated on to six monolayers, two of which were stained by each method. The average number of inclusions seen per monolayer was calculated from the numbers counted in at least 30 microscope fields ( $\times 500$ magnification) on each of the duplicates. To mimic clinical specimens containing few infective particles, a number of dilutions of FKC agent were each inoculated on to $10 \mathrm{McCoy}$ cell monolayers, five of these were stained with MG-NR and five with Giemsa. Each monolayer was examined (at $\times 200$ magnification) by two observers on a double blind basis.

Clinical specimens were each inoculated on to two monolayers, one of which was stained with MG-NR and the other using either Giemsa or FA. The monolayers were coded and examined (at $\times 200$ magnification) for the presence of inclusions by two observers on a double blind basis.

\section{Results}

No significant difference was observed between the numbers of inclusions counted ( $\times 500$ magnification) using the MG-NR, methylene blue and Giemsa methods for the eight different chlamydial strains tested (Table 1) $(p>0.05)$ but when specimens containing few infectious $C$ psittaci particles were used and monolayers were examined by low power microscopy ( $\times 200$ magnification), MG-NR was superior to Giemsa staining (Table 2).

Table 1 Average numbers of inclusions detected in McCoy cell monolayers inoculated with chlamydial isolates after staining with Giemsa, methylene blue or methyl green-neutral red

\begin{tabular}{llll}
\hline Chlamydia & Giemsa & Methylene blue & $\begin{array}{c}\text { Methyl green } \\
\text {-neutral red }\end{array}$ \\
\hline FKC & $1.1 \times 10^{3}$ & $4.2 \times 10^{3}$ & $6.6 \times 10^{3}$ \\
GPIC & $2.5 \times 10^{3}$ & $2.0 \times 10^{3}$ & $2.3 \times 10^{3}$ \\
IOL 207 & $1.4 \times 10^{4}$ & $1.5 \times 10^{4}$ & $1.5 \times 10^{4}$ \\
33L & $4.7 \times 10^{3}$ & $4.8 \times 10^{3}$ & $7.5 \times 10^{3}$ \\
EA & $1.8 \times 10^{3}$ & $1.8 \times 10^{3}$ & $1.8 \times 10^{3}$ \\
MPV & $5.0 \times 10^{3}$ & $5.3 \times 10^{3}$ & $5.7 \times 10^{3}$ \\
395 & $1.0 \times 10^{3}$ & $1.3 \times 10^{3}$ & $1.7 \times 10^{5}$ \\
Cal-15 & $2.2 \times 10^{3}$ & $1.6 \times 10^{3}$ & $1.3 \times 10^{3}$ \\
Cal-15* & $2.2 \times 10^{3}$ & $2.6 \times 10^{3}$ & $2.4 \times 10^{3}$ \\
\hline
\end{tabular}

*Inclusions counted by dark field illumination.

Table 2 Comparison of methyl green-neutral red and Giemsa staining for the detection of FKC agent inclusions in specimens with low infectivity titres

\begin{tabular}{|c|c|c|c|c|}
\hline \multirow[t]{3}{*}{ Dilution } & \multicolumn{4}{|c|}{ No of positive monolayers } \\
\hline & \multicolumn{2}{|c|}{ Methyl green-neutral red } & \multicolumn{2}{|l|}{ Giemsa } \\
\hline & Observer 1 & Observer 2 & Observer 1 & Observer 2 \\
\hline $10^{-4}$ & $5 / 5$ & $5 / 5$ & $0 / 5$ & $0 / 5$ \\
\hline $10^{-5}$ & $3 / 5$ & $4 / 5$ & $0 / 5$ & $0 / 5$ \\
\hline $10^{-6}$ & $2 / 5$ & $1 / 5$ & $0 / 5$ & $0 / 5$ \\
\hline $10^{-7}$ & $0 / 5$ & $1 / 5$ & $0 / 5$ & $0 / 5$ \\
\hline
\end{tabular}

For the $C$ psittaci strains, the MG-NR method produced clearly stained inclusions which were well differentiated and were easily detected by bright field illumination in every case. In our hands, the differentiation achieved with methylene blue appeared in general to offer little advantage over Giemsa. In the case of the $C$ trachomatis strain, MG-NR stained inclusions (pale pink) were poorly differentiated from the background when viewed by 
bright field illumination, but could be distinguished easily when dark field illumination was used.

Of 96 conjunctival swabbings taken from guinea pigs and rabbits experimentally infected with FKC agent, $66(69 \%)$ were positive by MG-NR, but only $56(58 \%)$ by Giemsa. Three specimens were only positive by Giemsa and 13 specimens were positive by MG-NR alone. The difference between these two methods was statistically significant $(\mathrm{p}<0.05)$.

A separate series of 66 conjunctival and urethral swabbings taken from guinea pigs inoculated experimentally with GPIC agent was used to compare the MG-NR staining method with FA staining; $35(53 \%)$ were positive by MG-NR and $37(56 \%)$ by FA. Five specimens were positive by FA alone while three were positive only by MG-NR. The difference between the results for these two methods was not significant $(p>0.05)$.

\section{Discussion}

Chlamydia psittaci infections occur commonly in animals and are often transmitted to humans to cause disease. Isolation in tissue culture has not been widely used for the diagnosis of these diseases, possibly because the inclusion bodies formed in cell culture monolayers by many of these organisms cannot be reliably detected by the Giemsa or iodine staining methods which have been used routinely for $C$ trachomatis. ${ }^{3} 4$

The results of this study show that the MG-NR staining method is more sensitive than Giemsa and is comparable in sensitivity to FA staining for detecting $C$ psittaci inclusions. In addition, $C$ trachomatis inclusions could be readily detected by dark field microscopy, thereby allowing the staining method to be used for detecting both $C$ psittaci and $C$ trachomatis inclusions. It is quick and easy to do, producing slides ready for examination in about $\mathbf{3 0}$ min, which is shorter than the time needed for either Giemsa or indirect immunofluorescence. The greater contrast achieved by the MG-NR method produced an additional saving in time in that monolayers could be examined rapidly at low magnification. It has proved to be suitable for routine use for the detection of both species of chlamydiae; examination being carried out by bright field or dark field illumination, or both, as required.

As part of the MG-NR staining procedure, an acid (pH 5.0) differentiation stage was used to increase the contrast between the inclusions and the cells. Acid conditions have been used at some stage in many of the staining procedures which have been described for chlamydiae ${ }^{369}$ but the importance of this does not appear to have been emphasised. In addition to MG-NR, we have noticed that many other dyes, such as Giemsa, May-Grünwald stain, malachite green, methylene blue, and toluidine blue, gave a much better contrast between inclusions and cells when used at $\mathrm{pH} 5.0$ than at the standard $\mathrm{pH}$ 6.8 to $7 \cdot 2$ which is recommended for Giemsa by the World Health Organisation. ${ }^{10}$

The authors wish to thank Mr R Hejazi and Mrs M Pink for assistance with clinical specimens. This work was supported by grants from the Department of Health and Social Security, an anonymous donor, and the Locally Organised Research Scheme, Moorfields Eye Hospital.

\section{References}

${ }^{1}$ Hanna L. Microscopic demonstration of chlamydial inclusions by Giemsa, iodine, or immunofluorescence stains. In: Hobson D, Holmes KK, eds. Non gonococcal urethritis and related infections. Washington DC: American Society for Microbiology, 1977:226-71.

2 Darougar S, Woodland RM, Forsey T, Cubitt S, Allami J, Jones BR. Isolation of Chlamydia from ocular infections. In: Hobson D, Holmes KK, eds. Non-gonococcal urethritis and related infections. Washington DC: American Society for Microbiology, 1977:295-8.

${ }^{3}$ Johnson FWA, Chancerelle LYJ, Hobson D. An improved method for demonstrating the growth of chlamydiae in tissue culture. Med Lab Sci 1978;35:67-74.

${ }^{4}$ Woodland RM, El-Sheikh H, Darougar S, Squires S. Sensitivity of immunoperoxidase and immunofluorescence staining for detecting chlamydia in conjunctiva scrapings and in cell culture. J Clin Pathol 1978;31: 1073-7.

${ }^{5}$ Gordon FB, Quan AL. Occurrence of glycogen in inclusions of psittacosis-lymphogranuloma venereumtrachoma agents. $J$ Infect Dis 1965;115:186-96.

6 Johnson FWA. A comparison of staining techniques for demonstrating group A chlamydia in tissue culture. Med Lab Technol 1975;32:233-8.

7 Dwyer RStC, Treharne JD, Jones BR, Herring J. Chlamydial infection: Results of micro-immunofluorescence tests for the detection of type-specific antibody in certain chlamydial infections. $\mathrm{Br} J$ Vener Dis $1972 ; 48$ : 452-9.

${ }^{8}$ Ripa KV, Mardh PA. New simplified culture technique for Chlamydia trachomatis. In: Hobson D, Holmes KK, eds. Non-gonococcal urethritis and related infections. Washington DC: American Society for Microbiology, 1977;323-7.

${ }^{9}$ Reeve P, Taverne J. A simple method for total particle counts of trachoma and inclusion blennorrhoea viruses. Nature 1962;195:923-4.

${ }^{10}$ World Health Organisation. Guide to the diagnosis of trachoma. Geneva, 1975.

Requests for reprints to: Dr RM Woodland, Subdepartment of Virology, Institute of Ophthalmology, Judd Street, London WC1H 9QS, England. 